\title{
Note on the stability for linear systems of differential equations
}

\author{
Qusuay H. Alqifiary ${ }^{1,2}$ \\ 1 University of Belgrade, Belgrade, Serbia \\ 2 University Of Al-Qadisiyah, Al-Diwaniya, Iraq \\ E-mail: qhaq2010@gmail.com
}

Copyright (C)2014 Qusuay.H. Alqifiary. This is an open access article distributed under the Creative Commons Attribution License, which permits unrestricted use, distribution, and reproduction in any medium, provided the original work is properly cited.

\begin{abstract}
In this paper, by applying the fixed point alternative method, we give a necessary and sufficient condition in order that the first order linear system of differential equations $\dot{z}(t)+A(t) z(t)+B(t)=0$ has the Hyers-Ulam-Rassias stability and find Hyers-Ulam stability constant under those conditions. In addition to that we apply this result to a second order differential equation $\ddot{y}(t)+f(t) \dot{y}(t)+g(t) y(t)+h(t)=0$.
\end{abstract}

Keywords: Fixed point method; Hyers-Ulam-Rassias stability; System of differential equations.

\section{Introduction}

In 1940, Hyers-Ulam stability was introduced by S.M. Ulam [18] to raise the question: suppose one has a function $y(t)$ which is close to solve an equation. Is there an exact solution $x(t)$ of the equation which is close to $y(t)$ ? (See $[4,23])$. In 1941, D.H. Hyers [4] gave an affirmative answer to the equation of Ulam for additive Cauchy equation in Banach spaces. A generalized solution to Ulam's problem for approximately linear mappings was proved by Th.M. Rassias in 1978. Th.M. Rassias [21] considered a mapping $f: E_{1} \rightarrow E_{2}$ such that $t \mapsto f(t x)$ is continuous in $t$ for each fixed $x$. Assume that there exists $\theta \geq 0$ and $0 \leq p<1$ such that

$$
\|f(x+y)-f(x)-f(y)\| \leq \theta\left(\|x\|^{p}+\|y\|^{p}\right)
$$

for any $x, y \in E_{1}$.

After that, many mathematicians have extended Ulam's problem in various directions (see $[5,11,13,14,19,20$, 22]). A Hyers-Ulam-Rassias problem with differential equations: The differential equation $\phi\left(f, y, y^{\prime}, \ldots, y^{(n)}\right)=0$ has the Hyers-Ulam-Rassias stability with respect to function $\theta$ if there exist a constant $M>0$ such that for given a function $y$ such that $\left|\phi\left(f, y, y^{\prime}, \ldots, y^{(n)}\right)\right| \leq \theta(t)$, there exists a solution $y_{c}$ of the differential equation such that $\left|y(t)-y_{c}(t)\right| \leq M \theta(t)$. Definition of Hyers-Ulam-Rassias stability significance since it means that if one is studying an Hyers-Ulam-Rassias stable system then one does not have to reach the exact solution.(which usually is quite difficult or time consuming). This is quite useful in many applications e.g. numerical analysis ,optimization,biology and economics etc., where finding the exact solution is quite difficult.

Jung has proved the Hyers-Ulam stability of linear differential equations (see [15, 16, 17]). Furthermore, Jung has also proved the Hyers-Ulam stability of linear differential equations by using the Laplace transform method (see $[7])$. 
The basic statements of the theory of ordinary differential equations (see $[1,2,3,6,8,10,12]$ ).

In this paper we will study stability of systems of first order with continuous coefficients, enabling us to apply the results to all differential equations of high order by transfering it to system of first order, and also apply to differential equations with constant coefficient in the same sense of proofs. There are two main sections : in section 3 , we will give a necessary and sufficient condition in order that the first order linear system of differential equations $\dot{z}(t)+A(t) z(t)+B(t)=0$ has the Hyers-Ulam-Rassias stability and find Hyers-Ulam stability constant under those conditions. In addition to that in section 4 , we will apply this result to a second order differential equation $\ddot{y}(t)+f(t) \dot{y}(t)+g(t) y(t)+h(t)=0$.

\section{Preliminaries and Auxiliary Results}

Definition 2.1 [5],[11],[22] Let I be any interval,let $z: I \rightarrow R^{n}, A: I \rightarrow R^{n \times n}, B: I \rightarrow R^{n}$, then

$\dot{z}(t)+A(t) z(t)+B(t)=0$

is Hyers-Ulam-Rassias stable with respect to $\varphi: I \rightarrow[0, \infty)$, with, $\|z(t)\|=\sum_{i=1}^{n}\left|z_{i}(t)\right|$, if there exists a real constant $K>0$ such that for each solution $s \in C^{1}\left(I, R^{n}\right)$ of inequality

$$
\|\dot{z}(t)+A(t) z(t)+B(t)\| \leq \psi(t)
$$

there exists a solution $z \in C^{1}\left(I, R^{n}\right)$ of equation (1) with

$$
\|s(t)-z(t)\| \leq K \varphi(t), \forall t \in I \text {. }
$$

Definition 2.2 For a nonempty set $X$, a function $d: X \times X \rightarrow[0, \infty]$ is called a generalized metric on $X$ if and only if d satisfies :

(i) $d(x, y)=0$ if and only if $x=y$;

(ii) $d(x, y)=d(y, x)$ for all $x, y \in X$

(iii) $d(x, z) \leq d(x, y)+d(y, z)$ for all $x, y, z \in X$.

Theorem 2.3 (The fixed point alternative) [9] Let $(X, d)$ be a generalized complete metric space. Assume that $\Lambda: X \rightarrow X$ is a strictly contractive operator with Lipschitz constant $L<1$. If there exists a nonnegative integer $k$ such that $d\left(\Lambda^{k+1} x, \Lambda^{k} x\right)<\infty$ for some $x \in X$, then the followings are true:

(a) The sequence $\left\{\Lambda^{n} x\right\}$ convergens to a fixed point $x^{*}$ of $\Lambda$;

(b) $x^{*}$ is the unique fixed point of $\Lambda$ in

$$
X^{*}=\left\{y \in X / d\left(\Lambda^{k} x, y\right)<\infty\right\}
$$

(c) If $y \in X^{*}$, then $d\left(y, x^{*}\right) \leq \frac{1}{1-L} d(\Lambda y, y)$.

Lemma 2.4 For given real numbers $a$ and $b$ with $a<b$, let $I=[a, b]$ be a closed interval and let be $X=\left\{f: I \rightarrow R^{n}\right.$, is continuous function $\}$, and $\mathrm{d}: \mathrm{X} \times \mathrm{X} \rightarrow[0, \infty]$ a function is defined as follows :

$$
d(f, g)=\inf \{c \in[0, \infty] /\|f(t)-g(t)\| \leq c \varphi(t) \forall t \in I\}
$$

where $\varphi(t): I \rightarrow(0, \infty)$ is a continuous function, then $d$ is a generalized metric on $X$.

Proof. By definition of a function d, then for all $f, g \in X$

(1) $d(f, g)=0 \leftrightarrow f(t)=g(t)$ for all $t \in I$;

(2) $d(f, g)=d(g, f)$. 
To prove that $d(f, g) \leq d(f, h)+d(h, g)$ for all $f, g, h \in X$.

Assume that $d(f, g)>d(f, h)+d(h, g)$ for some $f, h, g \in X$. Then, by definition of d, there exists $t_{0} \in I$ with

$$
\begin{aligned}
\left\|f\left(t_{0}\right)-g\left(t_{0}\right)\right\| & >\{d(f, h)-d(h, g)\} \varphi\left(t_{0}\right) \\
& =d(f, h) \varphi\left(t_{0}\right)-d(h, g) \varphi\left(t_{0}\right) \\
& \geq\left\|f\left(t_{0}\right)-h\left(t_{0}\right)\right\|+\left\|h\left(t_{0}\right)-g\left(t_{0}\right)\right\|
\end{aligned}
$$

and this is contradiction .

Lemma 2.5 For given real numbers $a$ and $b$ with $a<b$, let $I=[a, b]$ be a closed interval and let

$X=\left\{f: I \rightarrow R^{n}\right.$, is continuous function $\}$, consider a generalized metric function on $X, \mathrm{~d}: \mathrm{X} \times \mathrm{X} \rightarrow[0, \infty]$ which is defined as follows :

$d(f, g)=\inf \{c \in[0, \infty] /\|f(t)-g(t)\| \leq c \varphi(t) \forall t \in I\}$

where $\varphi(t): I \rightarrow(0, \infty)$ is a continuous function, then $(X, d)$ is a complete metric space.

Proof. Let $\left\{h_{n}\right\}$ be a Cauchy sequence in (X,d). Then $\forall \varepsilon>0$ there exist $N_{\varepsilon} \in N$ such that $d\left(h_{m}, h_{n}\right) \leq \varepsilon$ $\forall m, n \geq N_{\varepsilon}$.

That means that with equation (2)

$\forall \varepsilon>0 \exists N_{\varepsilon} \in N: \forall m, n \geq N_{\varepsilon}, \forall t \in I\left\|h_{m}(t)-h_{n}(t)\right\| \leq \varepsilon \varphi(t)$.

If $\mathrm{t}$ is fixed, equation (3) implies that $\left\{h_{n}(t)\right\}$ is a cauchy sequence in $R^{n}$. Since $R^{n}$ is complete, $\left\{h_{n}(t)\right\}$ converge for each $t \in I$. Thus, we can define a function $h: I \rightarrow R^{n}$ by $h(t)=\lim _{n \rightarrow \infty} h_{n}(t)$.

If we let $m \rightarrow \infty$, it then follows from (3) that

$\forall \varepsilon>0 \exists N_{\varepsilon} \in N: \forall n \geq N_{\varepsilon}, \forall t \in I\left\|h(t)-h_{n}(t)\right\| \leq \varepsilon \varphi(t)$,

that is, since $\varphi$ is bounded on I, $\left\{h_{n}(t)\right\}$ converges uniformly to $h$. Hence, $h$ is continuous and $h \in X$.

If we consider equation (2) and (4), then we may conclude that

$$
\forall \varepsilon>0 \exists N_{\varepsilon} \in N: \forall n \geq N_{\varepsilon} d\left(h, h_{n}\right) \leq \varepsilon
$$

that is, the cauchy sequence $\left\{h_{n}(t)\right\}$ converge to $h$ in $(X, d)$. Hence, $(X, d)$ is complete.

\section{First order linear system of differential equations}

We will prove the Hyers-Ulam -Rassias stability for the equation (1) on the intervals $I=[a, b)$, where $-\infty<a<$ $b \leq \infty$.

Theorem 3.1 Let $A: I \rightarrow R^{n \times n}$ and $B: I \rightarrow R^{n}$ be continuous matrices functions and let for a positive constant $N$, such that $\|A(t)\| \geq N$ for all $t \in I$.

Assume that $\psi: I \rightarrow[o, \infty)$ is an integrable function with the property that there exists $P \in(0,1)$ such that

$\int_{a}^{t}\left\|A\left(t_{1}\right)\right\| \psi\left(t_{1}\right) d t_{1} \leq P \psi(t)$

for all $t \in I$. If a continuously differential function $z: I \rightarrow R^{n}$ verifies the relation:

$\|\dot{z}(t)+A(t) z(t)+B(t)\| \leq \psi(t)$

for all $t \in I$. Then there exists a unique solution $s: I \rightarrow R^{n}$ of the equation (1) which verifies the following relation:

$\|z(t)-s(t)\| \leq \frac{P}{N-N P} \psi(t)$

for all $t \in I$ and $s(a)=z(a)$. 
Proof. Let us consider the set $\Omega=\left\{h: I \rightarrow R^{n} / \mathrm{h}\right.$ is continuous and $\left.\mathrm{h}(\mathrm{a})=\mathrm{z}(\mathrm{a})\right\}$ with a function $d: \Omega \times \Omega \rightarrow$ $[0, \infty]$ defined on $\Omega$ as

$d\left(h_{1}, h_{2}\right)=d_{\varphi}\left(h_{1}, h_{2}\right)=\inf \left\{K>0,\left\|h_{1}(t)-h_{2}(t)\right\| \leq K \varphi(t), \forall t \in I\right\}$. By lemma (2.4) and Lemma (2.5), the $(\Omega, d)$ is generalized complete metric space. We define the operator $T: \Omega \rightarrow \Omega$,

$$
T h(t)=z(a)-\int_{a}^{t}\left(A\left(t_{1}\right) h\left(t_{1}\right)+B\left(t_{1}\right)\right) d t_{1}, t \in I
$$

for all $h \in \Omega$. Indeed $T h$ is a continuously differentable function on I, since A and B are continuous functions and $T h(a)=z(a)$. Now, let $h_{1}, h_{2} \in \Omega$.Then we have

$$
\begin{aligned}
\left\|T h_{1}(t)-T h_{2}(t)\right\| & =\left\|\int_{a}^{t} A\left(t_{1}\right)\left(h_{1}\left(t_{1}\right)-h_{2}\left(t_{1}\right) d t_{1}\right)\right\| \\
& \leq \int_{a}^{t}\left\|A\left(t_{1}\right)\right\|\left\|h_{1}\left(t_{1}\right)-h_{2}\left(t_{1}\right)\right\| d t_{1} \\
& \leq d\left(h_{1}, h_{2}\right) \int_{a}^{t} A\left(t_{1}\right) \psi\left(t_{1}\right) d t_{1} \\
& \leq P \psi(t) d\left(h_{1}, h_{2}\right) \forall t \in I .
\end{aligned}
$$

Therefore,

$d\left(T h_{1}(t)-T h_{2}(t)\right) \leq P d\left(h_{1}, h_{2}\right)$

Thus, the operator $\mathrm{T}$ is a contraction with the constant $\mathrm{P}$. By integrating the both sides of the relation (6) on $[a, t]$ we obtain

$\left\|z(t)-z(a)+\int_{a}^{t}\left(A\left(t_{1}\right) z\left(t_{1}\right)+B\left(t_{1}\right)\right) d t_{1}\right\| \leq \frac{P}{N} \psi(t)$ for all $t \in I$.

which means $d(z, T z) \leq \frac{P}{N} \psi(t)<\infty$. By the fixed point alternative (2.3)there exists an element $s=\lim _{n \rightarrow \infty} T^{n} z$ and $s$ is a unique fixed point of $T$. In the set $\Delta=\left\{h \in \Omega / d\left(T^{n_{0}} z, h\right)<\infty\right\}$.

It may be proved that $\Delta=\{h \in \Omega / d(z, h)<\infty\}$. Therefore, the set $\Delta$ is independent of $n_{0}$. To prove that the function $s$ is a solution to the equation (1), we derive, with respect to $t$, the both sides of the following relation

$$
s(t)=T s(t) \forall t \in I
$$

Thus,

$$
\dot{s}(t)=-A(t) s(t)-B(t)
$$

for all $t \in I$, which implies that the function $\mathrm{s}$ is a solution of the equation (1) and verifies the relation $s(a)=z(a)$. Applying the fixed point alternative again, we obtain $d(h, s) \leq \frac{1}{1-P} d(h, T h)$ for all $h \in \Delta$. Since $z \in \Delta$, we have

$d(z, S) \leq \frac{1}{1-P} d(z, T z) \leq \frac{P}{N(1-P)}$. Hence,

$$
\|z(t)-s(t)\| \leq \frac{P}{N(1-P)} \psi(t)
$$

for all $t \in I$. This inequality proves the relation (1) .

In the same manner it is possible to proved the following theorem of the Hyers-Ulam-Rassias stability of the equation (1) on the interval $J=(b, a]$, where $-\infty \leq b<a<\infty$.

Theorem 3.2 Let $A: J \rightarrow R^{n \times n}$ and $B: J \rightarrow R^{n}$ be continuous matrices functions and let for a positive constant $N$, such that $\|A(t)\| \geq N$ for all $t \in J$. 
Assume that $\psi: J \rightarrow[o, \infty)$ is an integrable function with the property that there exists $P \in(0,1)$ such that

$\int_{a}^{t}\left\|A\left(t_{1}\right)\right\| \psi\left(t_{1}\right) d t_{1} \leq P \psi(t)$

for all $t \in J$. If a continuously differential function $z: J \rightarrow R^{n}$ verifies the relation :

$\|\dot{z}(t)+A(t) z(t)+B(t)\| \leq \psi(t)$

for all $t \in J$, then there exists a unique solution $s: J \rightarrow R^{n}$ of the equation (1) which verifies the following relation:

$\|z(t)-s(t)\| \leq \frac{P}{N-N P} \psi(t)$

for all $t \in J$ and $s(a)=z(a)$.

The Hyers-Ulam Rassias stability equation (1) on $R$ will be proved by Theorem (3.1) and Theorem (3.2).

Corollary 3.3 Let $A: R \rightarrow R^{n \times n}$ and $B: R \rightarrow R^{n}$ be continuous matrices functions and let for a positive constant $N$, such that $\|A(t)\| \geq N$ for all $t \in R$. Assume that $\psi: R \rightarrow[o, \infty)$ is an integrable function with the property that there exists $P \in(0,1)$ such that

$\left|\int_{0}^{t}\left\|A\left(t_{1}\right)\right\| \psi\left(t_{1}\right) d t_{1}\right| \leq P \psi(t)$

for all $t \in R$. If a continuously differential function $z: R \rightarrow R^{n}$ verifies the relation :

$\|\dot{z}(t)+A(t) z(t)+B(t)\| \leq \psi(t)$

for all $t \in R$, then there exists a unique solution $s: R \rightarrow R^{n}$ of the equation (1) which verifies the following relation:

$\|z(t)-s(t)\| \leq \frac{P}{N-N P} \psi(t)$

for all $t \in R$ and $s(0)=z(0)$.

Proof. By relation (13) we have

$\int_{0}^{t}\left\|A\left(t_{1}\right)\right\| \psi\left(t_{1}\right) d t_{1} \leq P \psi(t)$

for all $t \geq 0$. Applying Theorem (3.1), there exists a solution of equation (1), $s_{1}:[0, \infty) \rightarrow R^{n}$, which verifies relations $(7)$ and $s_{1}(o)=z(o)$. From relation(13) we also obtain

$\int_{t}^{o}\left\|A\left(t_{1}\right)\right\| \psi\left(t_{1}\right) d t_{1} \leq P \psi(t)$

for all $t \leq 0$.Applying Theorem (3.2), there exists a solution of equation (1), $s_{2}:(-\infty, 0] \rightarrow R^{n}$ which verifies relation $(12)$ and $s_{2}(o)=z(o)$.It is easy to check if the function

$s(t)=\left\{\begin{array}{l}s_{1}(t), t \geq 0 \\ s_{2}(t), t<0\end{array}\right.$

is a continuously differentiable function on $R$, a solution of equation (1) on $R$ and it verifies relation (15). 
Corollary 3.4 Let $A \neq 0$ be $n \times n$ constant matrix and $B: R \rightarrow R^{n}$ be $n \times 1$ a continuous matrix function $(n \times 1$ constant matrix ). Assume that $\psi: R \rightarrow[o, \infty)$ is an integrable function with the property that there exists $P \in(0,1)$ such that

$\left|\int_{0}^{t} \psi\left(t_{1}\right)\right| \leq \frac{P}{\|A\|} \psi(t)$

for all $t \in R$. If a continuously differential function $z: R \rightarrow R^{n}$ verifies the relation:

$\|\dot{z}(t)+A z(t)+B(t)\| \leq \psi(t)(\|\dot{z}(t)+A z(t)+B\| \leq \psi(t))$

for all $t \in R$, then there exists a unique solution $s: R \rightarrow R^{n}$ of the equation

$$
\dot{z}(t)+A z(t)+B(t)=0(\dot{z}(t)+A z(t)+B=0)
$$

which verifies the following relation:

$\|z(t)-s(t)\| \leq \frac{P}{\|A\|(1-P)} \psi(t)$

for all $t \in R$ and $s(0)=z(0)$.

Proof. By Corollary (3.3) and let $N=\|A\|$.

\section{Second order differential equation}

In this section we will prove the Hyers-Ulam -Rassias stability for the following scallar equation

$\ddot{y}(t)+f(t) \dot{y}(t)+g(t) y(t)+h(t)=0$

In the same manner, at first we will prove the Hyers-Ulam -Rassias stability for the equation (22) on the intervals $I=[a, b)$, where $-\infty<a<b \leq \infty$.

Theorem 4.1 Let $f, g, h: I \rightarrow R$ be continuous functions and let for a positive constant $0<N<1$, $|f(t)|+|g(t)| \geq N$ for all $t \in I$. Assume that $\psi: I \rightarrow[o, \infty)$ is an integrable function with property that there exists $P \in(0,1)$ such that

$\int_{a}^{t}\left(1+\left|f\left(t_{1}\right)\right|+\left|g\left(t_{1}\right)\right|\right) \psi\left(t_{1}\right) d t_{1} \leq P \psi(t)$

for all $t \in I$. If a function $y \in C^{2}(I, R)$ verifies the relation

$|\ddot{y}(t)+f(t) \dot{y}(t)+g(t) y(t)+h(t)| \leq \psi(t)$

for all $t \in I$, then there exists a unique solution $s_{1}: I \rightarrow R$ of equation (22), which verifies the following relation

$\left|y(t)-s_{1}(t)\right| \leq \frac{P}{N(1-P)} \psi(t)$

for all $t \in I$, and $s_{1}(a)=y(a)$.

Proof. Let $z_{1}(t)=y(t), z_{2}(t)=\dot{z}_{1}(t), \dot{z}_{2}(t)=-f(t) z_{2}(t)-g(t) z_{1}(t)$.

Let $z(t)=\left[\begin{array}{l}z_{1}(t) \\ z_{2}(t)\end{array}\right], A(t)=\left[\begin{array}{cc}0 & -1 \\ g(t) & f(t)\end{array}\right], H(t)=\left[\begin{array}{c}0 \\ h(t)\end{array}\right]$, then equation(22) transfer into

$\dot{z}(t)+A(t) z(t)+H(t)=0$ 
for all $t \in I$. By hypotheses, since $|f(t)|+|g(t)| \geq N$, then $\|A(t)\|=1+|f(t)|+|g(t)| \geq N$ and by relation (23) we obtain $\int_{a}^{t}\|A(t)\| \psi\left(t_{1}\right) d t_{1} \leq P \psi(t)$.

Now, let a function y satisfy the relation (24), since

$$
\begin{aligned}
\mathrm{z}(\mathrm{t})=\left[\begin{array}{l}
z_{1}(t) \\
z_{2}(t)
\end{array}\right]=\left[\begin{array}{l}
y(t) \\
\dot{y}(t)
\end{array}\right] \text { and } & \\
\dot{z}(t)+A(t) z(t)+H(t) & =\left[\begin{array}{l}
\dot{y}(t) \\
\ddot{y}(t)
\end{array}\right]+\left[\begin{array}{cc}
0 & -1 \\
g(t) & f(t)
\end{array}\right]\left[\begin{array}{c}
y(t) \\
\dot{y}(t)
\end{array}\right]+\left[\begin{array}{c}
0 \\
h(t)
\end{array}\right] \\
& =\left[\begin{array}{c}
\dot{y}(t)-\dot{y}(t) \\
\ddot{y}(t)+f(t) \dot{y}(t)+g(t) y(t)+h(t)
\end{array}\right]
\end{aligned}
$$

$\Rightarrow\|\dot{z}(t)+A(t) z(t)+H(t)\|=|\ddot{y}(t)+f(t) \dot{y}(t)+g(t) y(t)+h(t)| \leq \psi(t)$, Hence, by Theorem (3.1), there exists solution such $s(t)=\left[\begin{array}{l}s_{1}(t) \\ s_{2}(t)\end{array}\right]$ of equation $(26)$ and $s(a)=z(a)$ such that $\|z(t)-s(t)\| \leq \frac{P}{N(1-P)} \psi(t)$ for all $t \in I$. Therefore, there exists $s_{1}(t)$ satisfying equation $(22)$ and $s_{1}(a)=y(a)$ such that $\left|y(t)-s_{1}(t)\right| \leq \frac{P}{N(1-P)} \psi(t)$ for all $t \in I$.

In the same manner and by Theorem (3.2) we can prove the Hyers-Ulam -Rassias stability for the equation (22) on the interval $J=(b, a]$, where $-\infty \leq b<a<\infty$.

Theorem 4.2 Let $f, g, h: J \rightarrow R$ be continuous functions and let for a positive constant $0<N<1$, $|f(t)|+|g(t)| \geq N$ for all $t \in J$. Assume that $\psi: J \rightarrow[o, \infty)$ is an integrable function with property that there exists $P \in(0,1)$ such that

$\int_{t}^{a}\left(1+\left|f\left(t_{1}\right)\right|+\left|g\left(t_{1}\right)\right|\right) \psi\left(t_{1}\right) d t_{1} \leq P \psi(t)$

for all $t \in J$. If a function $y \in C^{2}(J, R)$ verifies the relation

$|\ddot{y}(t)+f(t) \dot{y}(t)+g(t) y(t)+h(t)| \leq \psi(t)$

for all $t \in J$. then there exists a unique solution $s_{1}: J \rightarrow R$ of equation (22), which verifies the following relation

$\left|y(t)-s_{1}(t)\right| \leq \frac{P}{N(1-P)} \psi(t)$

for all $t \in J$, and $s_{1}(a)=y(a)$.

Now in the same manner Corollary (3.3) and by Theorems (4.1) and (4.2), we obtain the following Corollary

Corollary 4.3 Let $f, g, h: R \rightarrow R$ be continuous functions and let for a positive constant $0<N<1$,

$|f(t)|+|g(t)| \geq N$ for all $t \in R$. Assume that $\psi: R \rightarrow[o, \infty)$ is an integrable function with property that there exists $P \in(0,1)$ such that

$\left|\int_{0}^{t}\left(1+\left|f\left(t_{1}\right)\right|+\left|g\left(t_{1}\right)\right|\right) \psi\left(t_{1}\right) d t_{1}\right| \leq P \psi(t)$

for all $t$ in $R$. If a function $y \in C^{2}(R, R)$ verifies the relation

$|\ddot{y}(t)+f(t) \dot{y}(t)+g(t) y(t)+h(t)| \leq \psi(t)$

for all $t \in R$, then there exists a unique solution $s_{1}: R \rightarrow R$ of equation (22), which verifies the following relation

$\left|y(t)-s_{1}(t)\right| \leq \frac{P}{N(1-P)} \psi(t)$

for all $t \in R$, and $s_{1}(0)=y(0)$.

Remark 4.4 The results can be applied to all differential equations of higher order by transfering it to system of first order. 


\section{References}

[1] C.Alsina and R.Ger, On some inequalities and stability results related to the exponential function, J.of Inequal and Appl.. 2, (1998),373-380.

[2] C. and Chicone, Ordinary differential equations with applications, Springer,New York, (2006).

[3] C. and Corduneanu, Principles of differential and integral equations, Chelsea Publ.Company,New York,(1971).

[4] D. H. Hyers, On the stability of the linear functional equation, Proc. Natl. Soc. USA 27 (1941), $222-224$.

[5] D. Popa, Hyers-Ulam-Rassias stability of a linear recurrence, J. Math. Anal. Appl. 309 (2005), 591-597.

[6] H. and Amann, Ordinary differential equations, Walter de Gruyter,Berlin , (1990) .

[7] H. Rezaei, S.-M. Jung and Th. M. Rassias, Laplace transform and Hyers-Ulam stability of linear differential equations, J. Math. Anal. Appl. 403 (2013), 244-251.

[8] Hsu and ,S.-B., Ordinary differential equations with applications, World Scientific,New Jersey, (2006).

[9] J.B.Diaz and B.Margolis, A fixed point theorem of the alternative for contractions on a generalized complete metric space,Bull.Amer.Math.Soc., 74, (1968), 305-309.

[10] J.Jost, Postmodern Analysis, Third Edition ,Springer (2005).

[11] P. Găvrută and L. Găvrută, A new method for the generalized Hyers-Ulam-Rassias stability, Internat. J. Nonlinear Anal. 2 (2010), 11-18.

[12] R. Bellman, Stability Theory of Differential Equations, New York (1953).

[13] S.-M. Jung, Hyers-Ulam stability of first order linear differential equations with constant coefficients ,Math.Anal.Appl., $320(2006), 549-561$.

[14] S.-M. Jung,and Rassias, Generalized Hyers-Ulam stability of Riccati differential equation,Math.Ineq.Appl., 11(2008),No.4,777-782.

[15] S.-M. Jung, Hyers-Ulam stability of linear differential equations of first order, Appl. Math. Lett. 17 (2004), $1135-1140$.

[16] S.-M. Jung, Hyers-Ulam stability of linear differential equations of first order, III, J. Math. Anal. Appl. 311 (2005), $139-146$.

[17] S.-M. Jung, Hyers-Ulam stability of linear differential equations of first order, II, Appl. Math. Lett. 19 (2006), 854-858.

[18] S. M. Ulam, Problems in Modern Mathematics, Chapter VI, Scince Editors, Wiley, New York, 1960.

[19] T.Mura,S-E.Takahasi,H.Choda, On the Hyers-Ulam stability for real continuous function valued differentiable map, Tokyo J.Math. 24, (2001),467-478.

[20] T.Mura,S-E.Takahasi,H.Choda, On the Hyers-Ulam stability for real continuous function valued differentiable map, Tokyo J.Math. 24, (2001),467-478.

[21] Th. M. Rassias, On the stability of the linear mapping in Banach spaces, Proc. Amer. Math. Soc. 72 (1978), 297-300.

[22] Y.-H. Lee and K.-W. Jun, A generalization of Hyers-Ulam-Rassias stability of Jensen's equation, J. Math. Anal. Appl. 238 (1999), 305-315.

[23] Y. Li and Y. Shen, Hyers-Ulam stability of nonhomogeneous linear differential equations of second order, Internat. J. Math. Math. Sci. 2009 (2009), Article ID 576852, 7 pages. 